\title{
A qualitative evaluation of a regional obesity prevention programme
}

\author{
G. Middleton, H. Rigby and D. Evans \\ Faculty of Health Life and Social Sciences, School of Sport, Coaching and Exercise Science, University of Lincoln, Brayford \\ Way, Lincoln, Lincolnshire, LN6 7TS, UK
}

Finding a useful evidence-base for existing obesity prevention programmes is problematic due to complex designs and validity issues in this 'real-life' environment ${ }^{(1)}$. It is necessary to consider alternative approaches for decision making in these population-based situations ${ }^{(1,2)}$. The use of 'stakeholder' opinion should be valued to coordinate suitable implementation and progress ${ }^{(1,2)}$. In this study a large multi-component health promotion programme, facilitated by a Primary Care Trust, is qualitatively evaluated. The programme aims to reduce the prevalence of obesity in a geographical area in which approximately 160000 people live. Interventions within the programme (42) are based on promoting healthy eating practices and increasing physical activity across all community settings, encompassing people of all ages. This evaluation specifically investigated the delivery and receipt of the programme, as perceived by the stakeholders: senior health officials (SHOs - management level NHS and local authority personnel); health professionals (HPs - public health nutritionists, health promotion specialists, local authority officers); and community participants (CPs - local people identified as key informants) who had administered and experienced the programme since conception; October 2006.

After a purposive sampling process, semi-structured focus groups (2) and semi-structured interviews (20) with three stakeholder groups were employed $^{(3)}$. Interviews were conducted with SHOs $(n 5)$ and CPs $(n$ 15). Focus groups were conducted with HPs $(n$ 11). All data were digitally recorded, transcribed verbatim and supported by additional field notes. NVivo (QSR v7) was used to manage the data analysis process. Data was analysed through a process of thematic content analysis ${ }^{(3)}$.

Findings highlighted key themes which impact upon the delivery and receipt of preventative services. Both SHOs and HPs felt that the programme had taken substantial time to 'capacity build' and there is a requirement for further integration of services with a clear strategic direction for the current practice to be maintained and developed. The CPs reported improvements in physical, psychological, emotional and social well-being. The Table below outlines participant suggestions from the three stakeholder groups.

\begin{tabular}{lll}
\hline \multicolumn{2}{l}{ Stakeholder suggestions for future consideration } & \\
\hline HPs & \multicolumn{1}{c}{ Senior HPs } & Community participants \\
\hline Mainstream funding for key positions & Develop widespread connections & Venue availability and access \\
Continuation of interventions & Strategic level influence & Continuation of interventions \\
Social marketing & Facilities to equip families & Information clarity \\
Engage specifically with parents & Community needs analysis & Intervention timing \\
\hline
\end{tabular}

The data collected from stakeholders in this evaluation can inform regional policy and ground-level delivery. This provides 'contextual relevant' information for the decision-making processes which enables the refinement of the obesity prevention programme ${ }^{(1)}$.

1. Swinburn B, Gill T \& Kumanyika S (2005) Obes Rev 6, 23-33.

2. Sallis J \& Glanz K (2009) Milbank $Q$ 87, 123-154.

3. Harris J, Gleason P, Sheean P et al. (2009) J Am Diet Assoc 109, 80-90. 